\title{
NUMERICAL MODELLING OF ADSORPTION OF METALLIC PARTICLES ON GRAPHITE SUBSTRATE VIA MOLECULAR DYNAMICS SIMULATION
}

\author{
H. RAFII-TABAR
}

Nano-Science Simulation Group, Centre for Numerical Modelling and Process Analysis School of Computing and Mathematical Sciences, University of Greenwich

Woolwich Campus, Wellington Street, London SE18 6PF, UK

A computer-based numerical modelling of the adsorption process of gas phase metallic particles on the surface of a graphite substrate has been performed via the application of molecular dynamics simulation method. The simulation relates to an extensive STM-based experiment performed in this field, and reproduces part of the experimental results. Both two-body and many-body inter-atomic potentials have been employed. A Morse-type potential describing the metal-carbon interactions at the interface was specifically formulated for this modelling. Intercalation of silver in graphite has been observed as well as the correct alignments of monomers, dimers and two-dimensional islands on the surface.

PACS numbers: 02.60.Cb, 07.05.Tp, 68.55.-a, 81.05.Tp

\section{Introduction}

The research into the nucleation and growth of nano-phase metallic thin films on inert substrates, such as graphite, via atomic vapour deposition has been the subject of renewed interest [1] owing to the catalytic properties of these materials $[2,3]$, and more generally the possibility of incorporating metal clusters as building blocks into the fabrication of nano-scale electronic (or photonic) devices such as sensors [4].

In a heterogeneous system, such as metal atoms adsorbed on a graphite substrate, a lattice misfit between the adlayer and the surface can promote two-dimensional terrace nucleations as well as the possible formation of low-dimensional clusters, such as the so-called atomic wires, at the surface steps. An understanding of the underlying atomic-level dynamics involved in these processes could therefore provide an opportunity for devising practical techniques for producing nano-structured quantum devices in a controlled way [1]. 
The formation of a nanoscopic defect-free thin film on a supporting substrate constitutes a highly complex vapour-to-solid phase transition phenomenon involving a two-stage mechanism of initial condensation of the adatoms from the vapour phase followed by their subsequent diffusion and growth into layered structures at elevated time scales [5]. Individual atoms, or their clusters, arriving at the surface may not be initially in thermal equilibrium with the substrate and can execute a variety of motions before their eventual capture by the surface leading to the formation of the first monolayer [6]. These atoms may migrate over the surface due to thermal activation and/or their own kinetic energy parallel to the surface, or re-evaporate and desorb back into the vapour phase, or nucleate into 2-D and 3-D local clusters during their finite residence time on the surface, or be captured by the already existing clusters, or attach to special defect sites such as kinks, steps and dislocations. The condensation process is essentially a competition between the adsorption and desorption equilibrium processes. The relative strengths of the interfacial and inter-adatom bondings, as well as the lateral stresses due to the lattice mismatch, determine the growth mode of a thin film. For instance, in the Volmer-Weber mode, displayed by many systems of metals growing on insulators including many metals on alkali halides, graphite and other compounds such as mica [5], small local clusters are nucleated directly on the substrate and then coalesce into larger islands by diffusion. This happens when the adatoms interact far more strongly among themselves than with the underlying substrate.

The most comprehensive experimental studies concerning the behaviour of small metallic clusters on highly oriented pyrolitic graphite (HOPG) are the STM-based experiments of Ganz et al. [7-9]. They observed the adsorption and surface diffusion of monomers, dimers, clusters, two- and three-dimensional islands of $\mathrm{Ag}, \mathrm{Au}, \mathrm{Cu}$ and $\mathrm{Al}$ atoms on HOPG using a STM configuration in UHV.

In this paper we report on our numerical modelling of the experiment reported in Ref. [9] employing the method of computer-based molecular dynamics (MD) simulation. We have selected, as an example, the adsorption process of $\mathrm{Ag}$ atoms on a graphite substrate. Our modelling can be easily extended to the other types of metal atoms considered in that experiment. We have followed closely the experimental technique of vapour deposition on a surface as employed in that experiment.

\section{Summary of the experimental results of Ganz et al. [9]}

The experiment was performed with a STM configuration consisting of a tungsten tip and operating either in a current-imagining or in a topographic-imaging mode. The graphite was chosen as the substrate material primarily because of the ease with which it could be cleaved, providing atomically flat planes over a rather significant microscopic area of micron size.

The substrate, prior to the deposition, was imaged to have a perfect surface free from defects such as grain boundaries or vacancies. Submonolayer amounts of metal particles, approximating to $1 \%$ of a monolayer, were vapour-deposited onto the substrate in a time interval of 0.1 to 1.0 seconds. This low coverage made possible the identification of isolated metal atoms, and their nano-sized clusters, on the surface against a background of clean graphite whose lattice parameter 

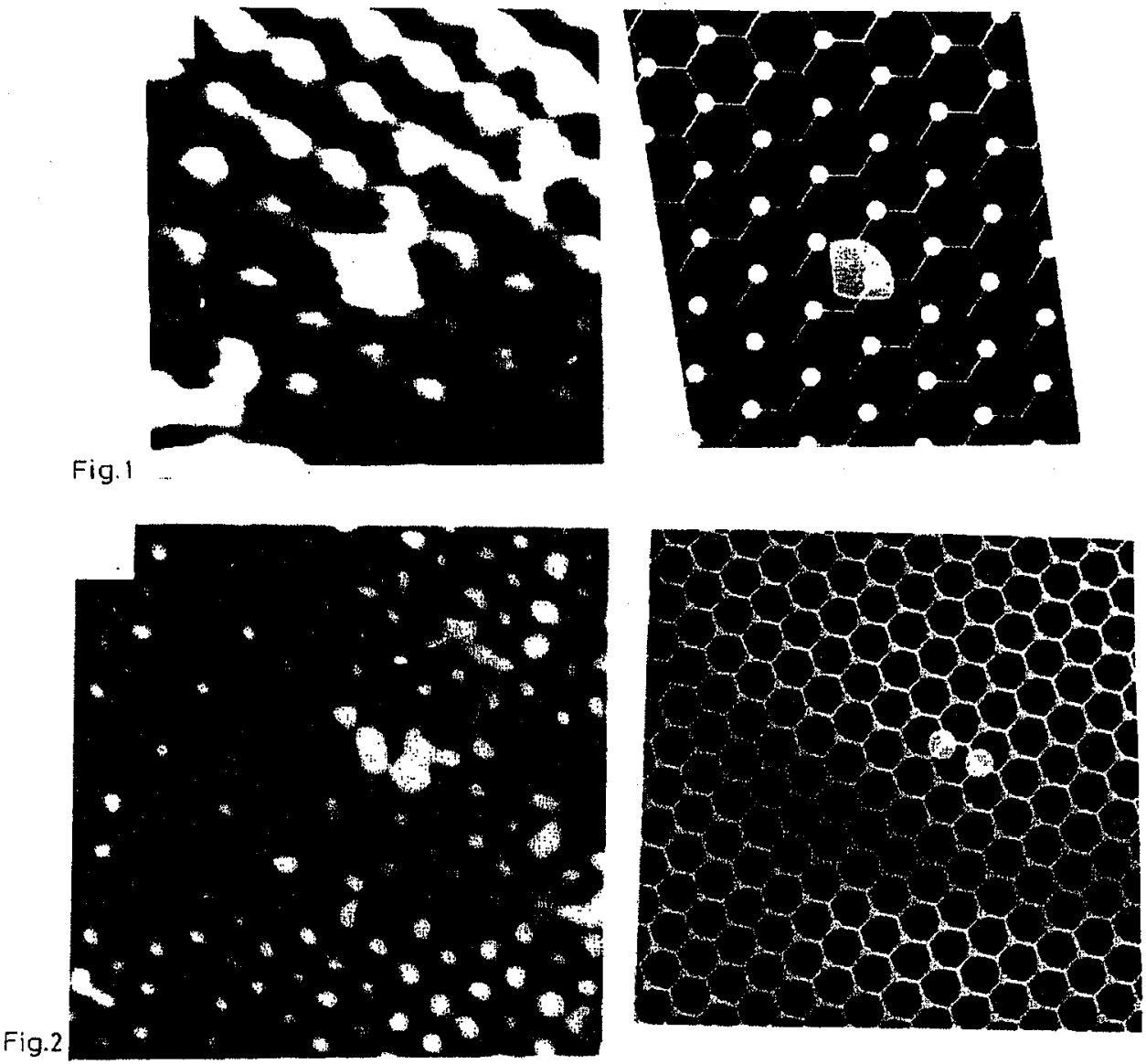

Fig. 1. A $14 \AA \times 16 \AA$ current image showing an isolated Ag monomer on graphite. On the right is a computer generated model showing the graphite lattice positions with the adatom shape superimposed. Figure reproduced from Ref. [9] with kind permission of Elsevier Science.

Fig. 2. A $32 \AA \times 32 \AA$ current image of an Ag dimer. The atoms are approximately at $\beta$-sites. Figure reproduced from Ref. [9] with kind permission of Elsevier Science.

( $a=2.46 \AA$ ) was used as an internal calibration measure to locate the adsorption sites by direct observation. The locations of these sites were determined via computer-generated topographical maps based on the digitization of observed locations of the graphite lattice spots and the adsorbed atoms obtained from the STM images.

On the basis of a set of STM images the following results relevant to the adsorption of $\mathrm{Ag}$ particles were reported:

Figures 1-3, taken from this experiment, show STM images, together with their computer-generated digitizations, of an isolated monomer, a dimer and a 

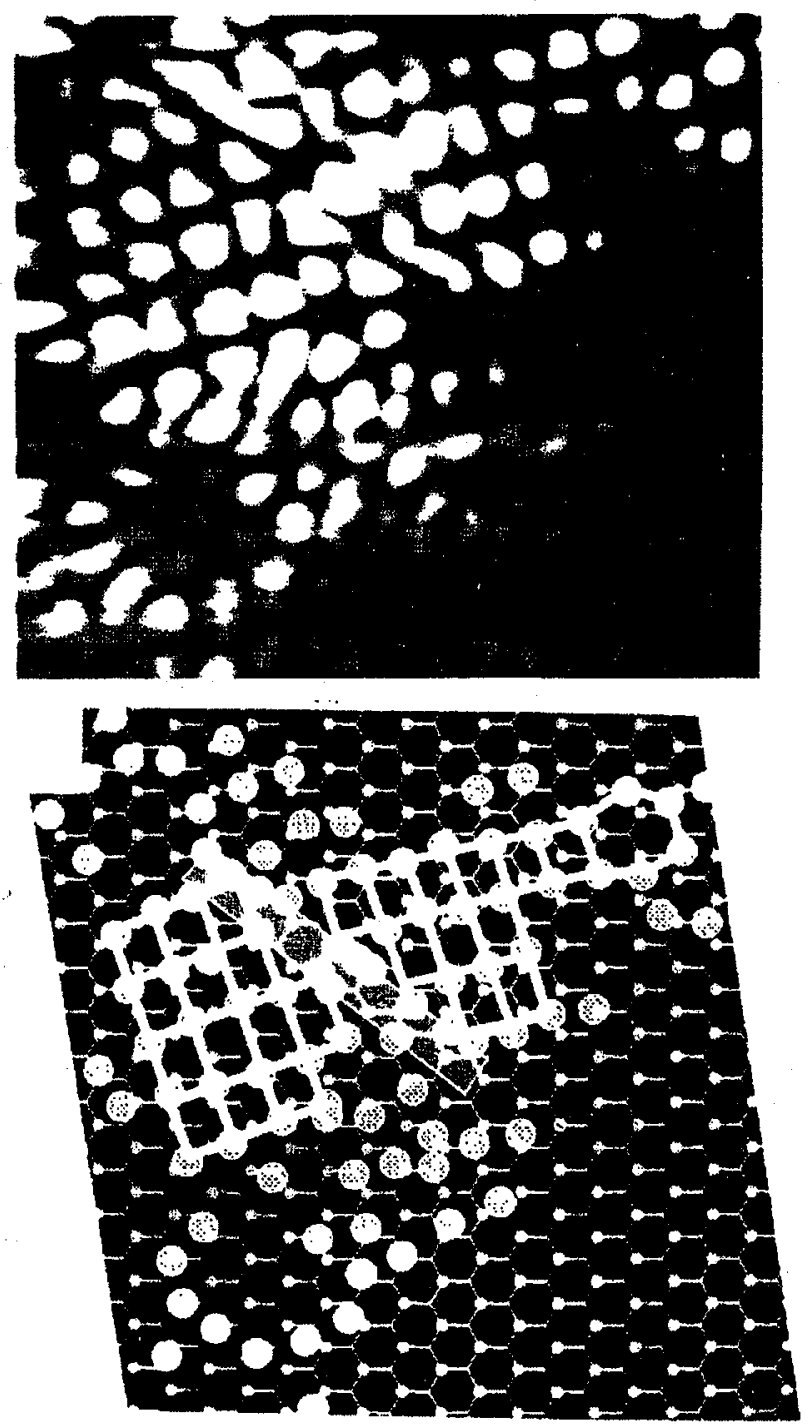

Fig. 3. A $35 \AA \times 35 \AA$ current image of a monolayer Ag island on graphite. The graphite honeycomb lattice is visible at the lower right. Lines on the computer generated model have been drawn to guide the eye. Filled circles are the positions of adatoms. Figure reproduced from Ref. [9] with kind permission of Elsevier Science.

small disordered island containing small ordered regions. The bright spots on each computer-generated figure represent the so-called $\beta$-sites on the graphite substrate. These are the sites with no atoms directly above or below in the adjacent basal planes. The monomer appeared to reside either on a $\beta$-site or on a bridge site 
connecting the first neighbours. No images of monomers above the hole sites were obtained. The dimer adatoms also resided at, or near, the $\beta$-sites with a bond length of $2.5 \pm 0.2 \AA$, comparable with a free dimer bond length of $2.50 \AA$.

The computer-generated model of the disordered island in Fig. 3 depicts the atoms as forming rectangular lattices that were neither commensurate with the substrate nor close-packed. A grain boundary can also be identified in the centre of the island.

\section{Molecular dynamics modelling}

\subsection{Phenomenological interatomic potentials}

In the present MD simulation, the energetics and dynamics of the overall adsorption process were obtained from a combination of four very different classical inter-atomic potentials, one of which, relevant to the interface $\mathrm{Ag}-\mathrm{C}$ interactions, was specifically constructed for this problem. Consequently the total interaction Hamiltonian of our system can be written as

$$
H_{\mathrm{I}}=H_{\mathrm{I}}^{\mathrm{Ag}-\mathrm{Ag}}+H_{\mathrm{I}}^{\mathrm{BC}-\mathrm{BC}}+H_{\mathrm{I}}^{\mathrm{NB}-\mathrm{NB}}+H_{\mathrm{I}}^{\mathrm{Ag}-\mathrm{C}},
$$

where the terms $H_{\mathrm{I}}^{\mathrm{Ag}-\mathrm{Ag}}, H_{\mathrm{I}}^{\mathrm{BC}-\mathrm{BC}}, H_{\mathrm{I}}^{\mathrm{NB}-\mathrm{NB}}$ and $H_{\mathrm{I}}^{\mathrm{Ag}-\mathrm{C}}$ refer, respectively, to the $\mathrm{Ag}-\mathrm{Ag}$ interactions, the $\mathrm{C}-\mathrm{C}$ covalent bonding within a basal plane hexagon, the $\mathrm{C}-\mathrm{C}$ van der Waals long-range interactions between the planes and the $\mathrm{Ag}-\mathrm{C}$ interactions at the interface.

\subsubsection{Ag-Ag long-range many-body potential}

The $H_{\mathrm{I}}^{\mathrm{Ag}-\mathrm{Ag}}$ term was modelled according to the long-range many-body interatomic potentials constructed by Sutton and Chen [10] for the atomistic modelling of the fcc elemental metals

$$
H_{\mathrm{I}}^{\mathrm{Ag}-\mathrm{Ag}}=\varepsilon\left[\sum_{i} \sum_{j>i} V^{\mathrm{Ag}-\mathrm{Ag}}\left(r_{i j}\right)-c Y^{\mathrm{Ag}} \sum_{i} \sqrt{\rho_{i}^{\mathrm{Ag}}}\right],
$$

where the specific forms of the various terms, as well as the potential parameters, are given in Ref. [10]. Both this Hamiltonian and its generalization [11] for the modelling of the fcc binary random alloys, have been used in the past in a number of large-scale MD simulations to model the indentation and adhesive properties of metallic nano-crystals using tip-substrate model geometries akin to those employed in the STM and the AFM configurations [12,13]. It should, however, be remarked that the Hamiltonian in Eq. (2) was originally constructed for the description of an ordered fcc lattice and its parameters were therefore obtained by fitting to such a morphology. Its application to an initially disordered system of Ag particles, such as the vapour phase in our model system, implied an approximation. However, our previous simulations $[12,13]$ show that for conditions where a metallic system has undergone a local melting transition the above potential still provided a reasonable description of the underlying dynamics. We did, however, find that a lower cut-off at $r_{\mathrm{c}}=0.57 a_{\mathrm{Ag}}$ was necessary for the correct modelling of this potential for the present high temperature simulation. 


\subsection{2. $C-C$ covalent bonding in a basal plane}

The $H_{\mathrm{I}}^{\mathrm{BC}-\mathrm{BC}}$ term was modelled via Tersoff non-central, bond-angle dependent many-body potential [14] as parameterized by Brenner [15, 16] to stabilize individual graphite planes and a diamond phase. The Tersoff potential models the total energy of a system of covalently-bonded atoms as a combination of pair-wise interactions with the coefficient of the attractive component (which acts as the bond order) depending on the local atomic environment and hence simulating the contribution of the many-body context to the dynamics of a particular atom. It has the form

$$
\begin{aligned}
& H_{\mathrm{I}}^{\mathrm{BC}-\mathrm{BC}}=\sum_{i} \mathrm{H}_{\mathrm{i}}^{\mathrm{BC}-\mathrm{BC}}= \\
& \quad \sum_{i} \sum_{j>i} f_{\mathrm{c}}\left(r_{i j}\right)\left[V_{R}^{\mathrm{BC}-\mathrm{BC}}\left(r_{i j}\right)-b_{i j} V_{A}^{\mathrm{BC}-\mathrm{BC}}\left(r_{i j}\right)\right],
\end{aligned}
$$

where $H_{i}$ represents the energy at site $i$, and the various terms are given in Ref. [14]. The function $f_{\mathrm{c}}$ is a switching function which restricts the pair interactions to the nearest neighbours, and $b_{i j}$ is the function that couples the $(i j)$ bond to the local many-body environment of the atom $i$.

\subsection{3. $C-C$ non-bonding potential between basal planes}

The $H_{\mathrm{I}}^{\mathrm{NB}-\mathrm{NB}}$ term was represented by the Lennard-Jones $(\mathrm{L}-\mathrm{J})$ potential [17] with parameters taken from a study of graphite [18]

$$
H_{\mathrm{I}}^{\mathrm{NB}-\mathrm{NB}}=4 \varepsilon \sum_{i} \sum_{j>i}\left[\left(\frac{\sigma}{r_{i j}}\right)^{12}-\left(\frac{\sigma}{r_{i j}}\right)^{6}\right] .
$$

To minimize the computational time, we applied a cut-off to this potential such that the atom $i$ in one basal plane interacted with those $j$ atoms in the next basal planes that were within a distance not greater than the inter-planar spacing, $c / 2$.

\subsubsection{Ag-C interface potential}

An interface potential forms the most significant component of the physics of any heterogeneous adsorption process. This controls the various stages of the growth process from the initial wetting of the substrate to the subsequent motions over the surface, and the eventual morphology of the emergent film.

The $H_{\mathrm{I}}^{\mathrm{Ag}-\mathrm{C}}$ term has, to our knowledge, never been formulated in the past, and we therefore had to develop an approximate scheme to obtain an analytic function for this terms. Such an approach was also reported recently for the description of $\mathrm{Pt}$ adsorption on graphite [19].

We adopted a Morse-type potential function

$$
H_{\mathrm{I}}^{\mathrm{Ag}-\mathrm{C}}=\sum_{i} \sum_{j>i} E_{\mathrm{Ag}-\mathrm{C}}\left[\mathrm{e}^{-N \delta\left(r_{i j}-r_{w}\right)}-N \mathrm{e}^{-\delta\left(r_{i j}-r_{w}\right)}\right]
$$

and to obtain its parameters, employed two known Morse potentials for the $\mathrm{C}-\mathrm{C}$ [20] and the $\mathrm{Ag}-\mathrm{Ag}$ [21] interactions

$$
\Phi_{\mathrm{I}}^{\mathrm{C}-\mathrm{C}}=\sum_{i} \sum_{j>i} E_{\mathrm{C}}\left[\mathrm{e}^{-2 \alpha_{1}\left(r_{i j}-r_{d}\right)}-2 \mathrm{e}^{-\alpha_{1}\left(r_{i j}-r_{d}\right)}\right]
$$


and

$$
\Phi_{\mathrm{I}}^{\mathrm{Ag}-\mathrm{Ag}}=\sum_{i} \sum_{j>i} E_{\mathrm{Ag}}\left[\mathrm{e}^{-m \alpha_{2}\left(r_{i j}-r_{0}\right)}-m \mathrm{e}^{-\alpha_{2}\left(r_{i j}-r_{0}\right)}\right] .
$$

A selection of mixing rules were then tried, and the following rule gave the best fit to the experimental results

$$
E_{\mathrm{Ag}-\mathrm{C}}=\sqrt{E_{\mathrm{C}} E_{\mathrm{Ag}}}, \quad r_{w}=\sqrt{r_{d} r_{0}}, \quad \delta=\sqrt{\alpha_{1} \alpha_{2}}, \quad N=\sqrt{2 m} .
$$

The parameters are given in Table.

\section{TABLE}

\begin{tabular}{l|l}
\multicolumn{2}{l}{ Parameters for $H_{1}^{\mathrm{Ag}-\mathrm{C}}$ potential. } \\
\hline \hline$\alpha_{1}=4.9519\left(\AA^{-1}\right)$ & $m=6.00$ \\
$\alpha_{2}=0.37152\left(\AA^{-1}\right)$ & $r_{0}=4.44476(\AA)$ \\
$E_{\mathrm{C}}=3.1(\mathrm{eV})$ & $r_{d}=1.2419(\AA)$ \\
$E_{\mathrm{Ag}}=0.0284875(\mathrm{eV})$ &
\end{tabular}

A modification which yields a zero value at the cut-off distance of $r_{\mathrm{c}}=2.5 a_{\mathrm{Ag}}$ was applied in accordance with the prescription given in Ref. [20]. A cut-off factor, given by

$$
h_{\mathrm{I}}^{\mathrm{Ag}-\mathrm{C}}(r)=H_{\mathrm{I}}^{\mathrm{Ag}-\mathrm{C}}\left(r_{\mathrm{c}}\right)+\left.S(r) \frac{\mathrm{d} H_{\mathrm{I}}^{\mathrm{Ag}-\mathrm{C}}}{\mathrm{d} r}\right|_{r=r_{\mathrm{c}}} \frac{1}{\left.\frac{\mathrm{d} S}{\mathrm{~d}}\right|_{r=r_{\mathrm{c}}}},
$$

where

$$
S(r)=1-e^{\eta\left(r-r_{c}\right)}
$$

was subtracted from (5) giving

$$
\begin{aligned}
& H_{\mathrm{I}}^{\mathrm{Ag}-\mathrm{C}}=\sum_{i} \sum_{j>i} E_{\mathrm{Ag}-\mathrm{C}}\left[\mathrm{e}^{-N \delta\left(r_{i j}-r_{w}\right)}-N \mathrm{e}^{-\delta\left(r_{i j}-r_{w}\right)}\right] \\
& -E_{\mathrm{Ag}-\mathrm{C}}\left[\mathrm{e}^{-N \delta\left(r_{\mathrm{c}}-r_{w}\right)}-N \mathrm{e}^{-\delta\left(r_{\mathrm{c}}-r_{w}\right)}\right] \\
& \quad-\frac{E_{\mathrm{Ag}-\mathrm{C} N \delta} N \delta}{\eta}\left[1-\mathrm{e}^{\eta\left(r_{i j}-r_{\mathrm{c}}\right)}\right]\left[\mathrm{e}^{-N \delta\left(r_{\mathrm{c}}-r_{w}\right)}-\mathrm{e}^{-\delta\left(r_{\mathrm{c}}-r_{w}\right)}\right] .
\end{aligned}
$$

The value $\eta=20$ was used. This was a sufficiently large value so that the function was only modified near the cut-off distance.

Employing the Hamiltonian in Eq. (1), the forces acting on individual atoms were calculated according to

$$
F_{i}=m_{i} a_{i}=\sum_{j>i} \frac{-\partial H_{\mathrm{I}}\left(r_{i j}\right)}{\partial r_{i j}} \widehat{r}_{i j} .
$$

These forces were then employed in the equations of motion based on the velocity Verlet algorithm [22]. 


\subsection{Temperature control}

In our modelling we dealt with two very different materials initially set at two widely different temperatures. While during the equilibration phase these two components had to be maintained at two different constant temperatures, during the adsorption phase the Ag atoms had to be cooled down continuously. The system had to be equilibrated for a second time when all the $\mathrm{Ag}$ atoms were adsorbed on the surface and reached its temperature. In such a complex situation a multi heat bath methodology can be set up so that different heat baths control different components of the system.

We implemented the temperature control by coupling a Nosé-Hoover $(\mathrm{N}-\mathrm{H})$ $[23,24]$ thermostat to the substrate atoms, whereas the $\mathrm{Ag}$ atoms were coupled to another, simpler, heat bath whereby their velocities were rescaled at every time step. We adopted this dual approach for the following reason.

The $\mathrm{N}-\mathrm{H}$ formalism couples an extra degree of freedom to the kinematics of the particles. This acts as a scaling variable and the particles are then either accelerated or decelerated depending on whether the time-dependent friction coefficient of the thermostat becomes respectively negative or positive. This implies that the system coupled to the thermostat is on average of the same temperature as the heat bath. Such a control was efficient for the substrate in our simulation since its temperature was always maintained at a constant value and hence the fluctuations were averaged out. For the Ag particles, we needed to cool them down by definite amounts and this meant rescaling their kinetic energies by definite amounts at each time step.

The implementation of the $\mathrm{N}-\mathrm{H}$ thermostat in a velocity Verlet formalism leads to substantial modifications of the equations of motion for the substrate particles, and these modified equations are given in Ref. [12].

\subsection{Adsorption process}

Our model system consisted of $200 \mathrm{Ag}$ atoms and 1920 carbon atoms in the substrate arranged in four graphite basal planes with 360, 480, 480 and 600 atoms, respectively, in the first, second, third and fourth planes. To mimic as closely as possible the experimental condition of atomic vapour deposition, the Ag particles were distributed randomly above the (0001) plane of the substrate. Figure $4 \mathrm{a}$ shows the initial configuration of the model system in the $\langle 100\rangle$ direction.

The initial temperature of the $\mathrm{Ag}$ vapour atoms was set at $2440 \mathrm{~K}$ [25] and that of the substrate at $300 \mathrm{~K}$. All the atoms were treated dynamically with periodic boundary conditions applied in the $x$ - and $y$-directions only. An equilibration run of 2500 time steps, at constant temperatures of $2440 \mathrm{~K}$ for the metal particles and $300 \mathrm{~K}$ for the substrate planes, with $\Delta t=3 \times 10^{-14}$ seconds, was performed during which the Ag particles were not allowed to interact with the substrate or with each other or to move in the $z$-direction. This lack of interaction between the vapour $\mathrm{Ag}$ particles ensured that no correlations were imposed among these particles. Both during the equilibration and following it the substrate was modelled as a rigid system whereby the atoms in each basal plane were subjected to an average force calculated for that basal plane from the forces experienced by all its atoms individually. This approximation ensured the structural stability of the substrate 


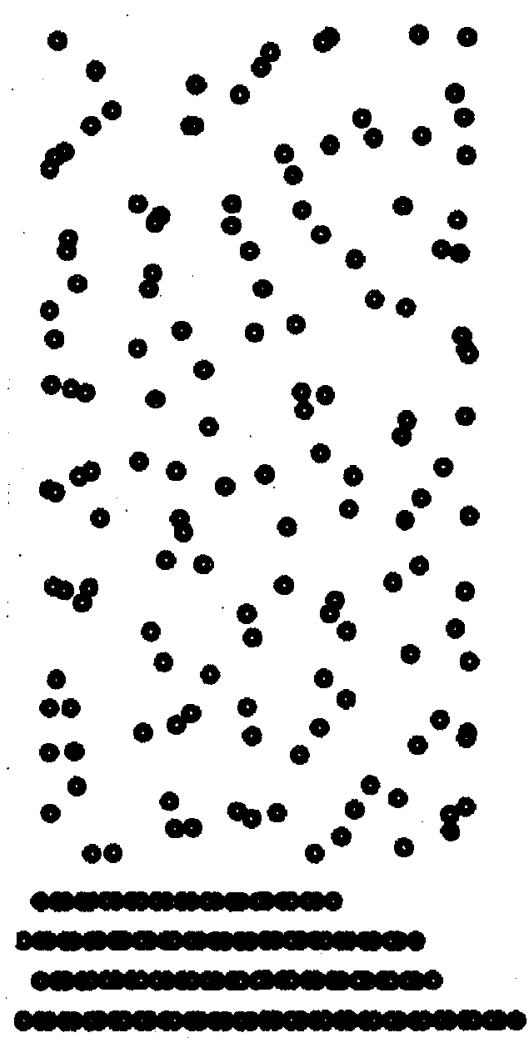

(a)

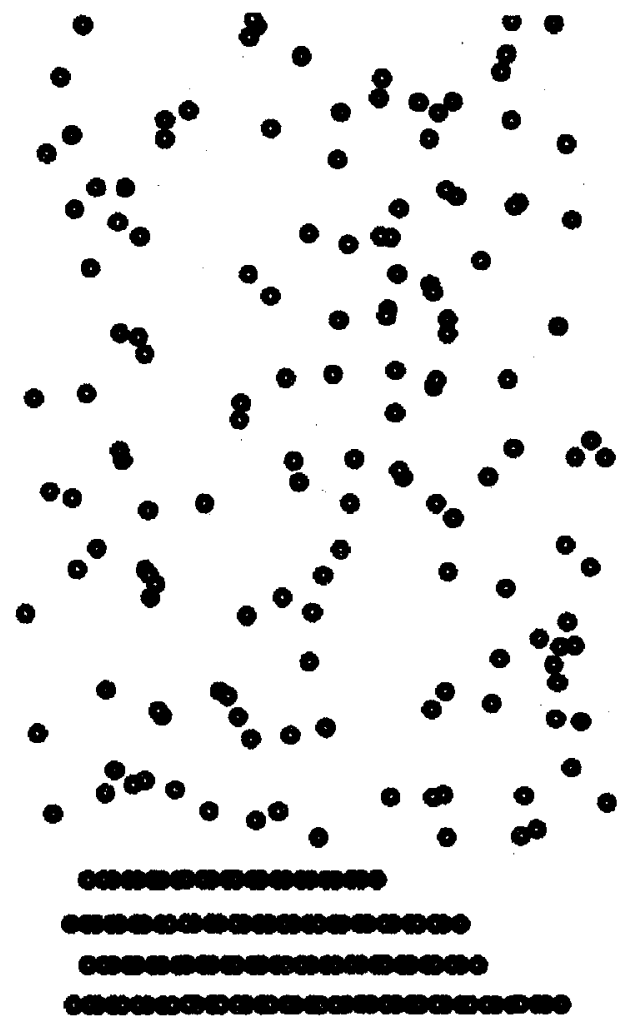

(b)

Fig. 4. (a) The initial configuration of the model system in $\langle 100\rangle$ direction showing randomly distributed $\mathrm{Ag}$ atoms over the graphite substrate. (b) The configuration of the system after equilibration for 2500 time steps.

system, preventing the local distortions of its geometry which would have made the task of identifying the final Ag alignments very difficult, if not impossible. Figure $4 \mathrm{~b}$ shows the state of the system after equilibration.

Following equilibration, the $\mathrm{Ag}$ particles were released in the $z$-direction of the substrate and a simulation run of 7500 time steps was performed. Again the $\mathrm{Ag}$ atoms were not allowed to interact with each other while in descent, ensuring that they arrived at the surface independently. To simulate the vapour deposition process, a negative uniform strain of $0.009 a_{\mathrm{Ag}}$ per time step was applied to every $\mathrm{Ag}$ atom along the $z$-direction. The strain was stopped once the $\mathrm{Ag}$ atoms were within a distance of $1.5 a_{\mathrm{Ag}}$ from the top layer of the substrate. Such a strain was equivalent to a drift velocity of $122.7 \mathrm{~m} \mathrm{~s}^{-1}$.

As the $\mathrm{Ag}$ atoms descended toward the substrate they were also being cooled down continuously at a rate of $0.34 \mathrm{~K}$ per time step. We chose this rather slow cooling rate so that the particles were still hot by the time they wetted the substrate. 
The cooling was continued till the Ag atoms attained a thermal equilibration with the substrate.

\section{Results and discussion}

Figure 5 shows the snapshots of the deposition process at various times. As the highly energetic $\mathrm{Ag}$ atoms approached the substrate, they penetrated the basal planes through the hexagons. This clearly represented the phenomenon of intercalation of $\mathrm{Ag}$ in HOPG. Three-dimensional animation of the run showed the $\mathrm{Ag}$ atoms executing very rapid bounce-on bounce-off motions between the basal planes before they were eventually captured by the surfaces. We believe that this could be a significant mechanism of capture of metallic particles by graphite.

The captured $\mathrm{Ag}$ atoms were still very mobile on the surface, nucleating and denucleating small 2-D and 3-D clusters as can be seen from Fig. 6, which shows different clusters on the second (0001) basal plane from the top. From this figure we can also see clearly that the Ag particles, on different basal planes, have displayed a Volmer-Weber growth mode.

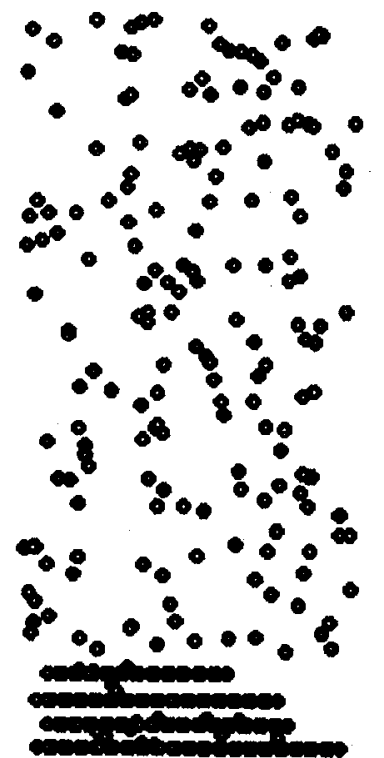

(a)

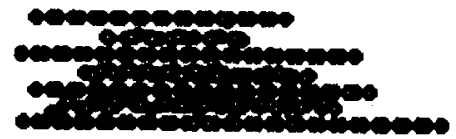

(d)

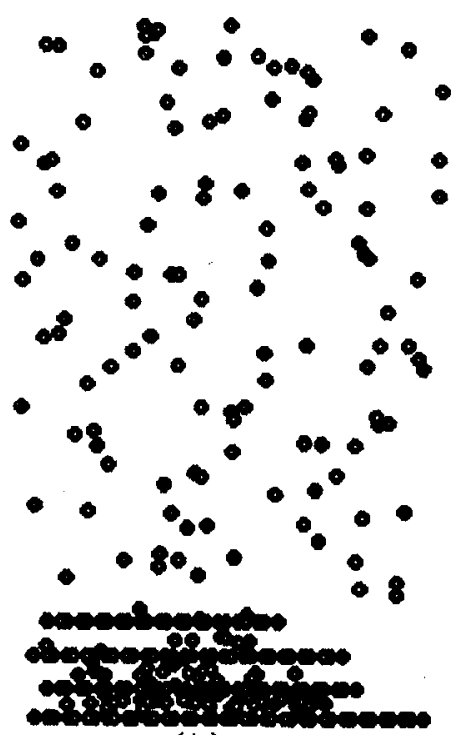

(b)

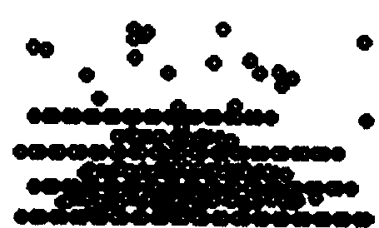

(c)

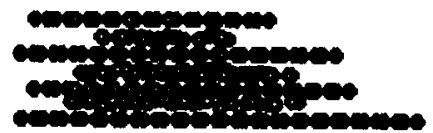

(e)

Fig. 5. A sequence of snapshots, along $\langle 100\rangle$ direction showing the deposition process after: (a) $7.5 \mathrm{ps}$, (b) $30 \mathrm{ps,} \mathrm{(c)} 75 \mathrm{ps}$, (d) $106 \mathrm{ps}$, (e) $180 \mathrm{ps}$ corresponding to Ag atoms' temperature of $2355 \mathrm{~K}, 2100 \mathrm{~K}, 1590 \mathrm{~K}, 1240 \mathrm{~K}$ and $400 \mathrm{~K}$, respectively. 


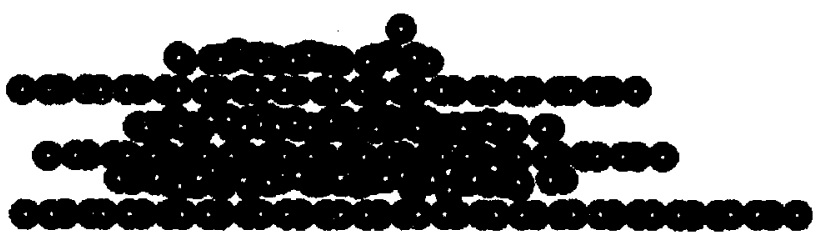

Fig. 6. A snapshot showing the formation of 2-D and 3-D clusters on the second (0001) basal plane from the top at $400 \mathrm{~K}$.

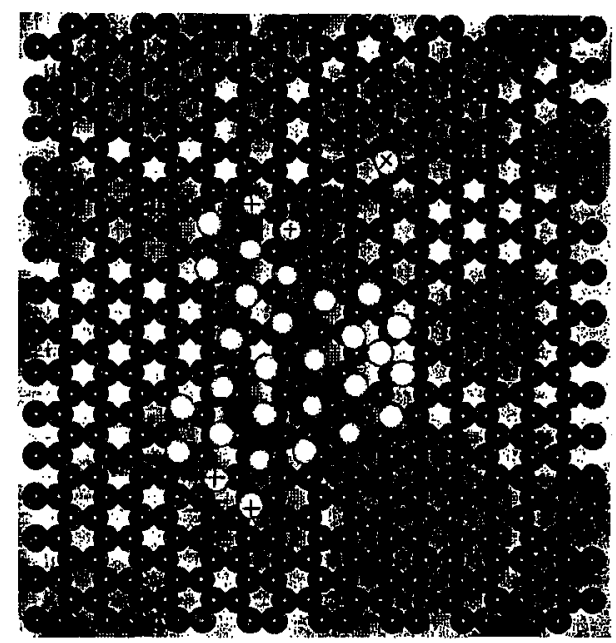

Fig. 7. The figure shows the final alignments of the Ag atoms on the second basal plane (0001) from the top, 1200 time steps after they first attained the substrate's temperature of $300 \mathrm{~K}$. An isolated monomer is shown with $(x)$ and a pair of dimers are indicated with ( + ). A grain boundary can also be observed in the centre of the island.

We also observed direct, but very transient, decoration of the steps. There were no diffusion-driven aggregation to the steps. One reason could be that the indirect decoration of steps, as seen experimentally [1], would have required terrace diffusion over elevated time scales of macroscopic dimensions.

The cooling of the $\mathrm{Ag}$ atoms to equilibrate with the substrate made them less mobile and more ordered. In Fig. 7 we show a snapshot of the $\mathrm{Ag}$ atoms 1200 time steps after they first equilibrated with the surface. A monomer and several dimers can be distinguished in this figure. It is evident that the monomer occupies a $\beta$-site. The two dimers also reside at or near the $\beta$-sites. These are in close agreement with the experimental findings [9] as depicted in Figs. 1 and 2. Our calculated dimmer bond length was $2.66 \AA$ which is comparable with $2.5 \pm 0.2 \AA$ reported in Ref. [9].

The general pattern of the $\mathrm{Ag}$ atoms on the lower right hand side of Fig. 7 shows a strong resemblance to the corresponding alignments in Fig. 3 for small $\mathrm{Ag}$ islands. We can observe the formation of rectangular lattices similar to those 
observed in the experiment, and also what appears to be a grain boundary. Similar patterns for the atoms on the other basal planes were identified as well.

In summary, our MD simulation, performed on a relatively small system and with the choice of several inter-atomic potentials, has been able to reproduce a portion of the experimental results in Ref. [9]. Simulations over longer time and with a much bigger number of particles would have improved the statistics of our results and could have led to the observation of the formation of nano-scale islands and their subsequent coalescence into a monolayer film.

\section{References}

[1] G.M. Francis, I.M. Goldby, L. Kuipers, B. von Issendroff, R.E. Palmer, J. Chem. Soc. Dalton Trans. 665 (1996).

[2] M. Boudart, Nature 372, 320 (1994).

[3] Z. Xu, F.S. Xiao, S.K. Purnell, O. Alexeev, S. Kawi, S.E. Deutsch, B.C. Gates, Nature 372, 357 (1994).

[4] W. Chen, H. Ahmed, K. Nakazato, Appl. Phys. Lett. 66, 3383 (1995).

[5] J.A. Venables, G.D.T. Spiller, M. Hanbucken, Rep. Prog. Phys. 47, 399 (1984).

[6] K.L. Chopra, Thin Film Phenomena, McGraw-Hill, London 1969.

[7] E. Ganz, K. Sattler, J. Clarke, J. Vac. Sci. Technol. A 6, 419 (1988).

[8] E. Ganz, K. Sattler, J. Clarke, Phys. Rev. Lett. 60, 1856 (1988).

[9] E. Ganz, K. Sattler, J. Clarke, Surf. Sci. 219, 33 (1989).

[10] A.P. Sutton, J. Chen, Philos. Magn. Lett. 61, 139 (1990).

[11] H. Rafii-Tabar, A.P. Sutton, Philos. Magn. Lett. 63, 217 (1991).

[12] A.P. Sutton, J.B. Pethica, H. Rafii-Tabar, J.A. Nieminen, in: Electron Theory in Alloy Design, Eds. D.G. Pettifor, A.H. Cottrell, Institute of Materials, London 1992, p. 191.

[13] H. Rafii-Tabar, Y. Kawazoe, Jpn. J. Appl. Phys. 32, 1394 (1993).

[14] J. Tersoff, Phys. Rev. B 39, 5566 (1989).

[15] D.W. Brenner, Mater. Res. Soc. Symp. Proc. 141, 59 (1989).

[16] D.W. Brenner, Phys. Rev. B 42, 9458 (1990).

[17] A. Cheng, M. Klein, Phys. Rev. B 45, 1889 (1992).

[18] W.A. Steele, The Interaction of Gases with Solid Surfaces, Pergamon Press, New York 1974.

[19] S.Y. Liem, K.-Y. Han, Surf. Sci. 328, 119 (1995).

[20] D.J. Oh, R.A. Johnson, Mater. Res. Soc. Symp. Proc. 141, 51 (1989).

[21] F. Milstein, J. Appl. Phys. 44, 3825 (1973).

[22] M.P. Allen, D.J. Tildesley, Computer Simulation of Liquids, Clarendon Press, Oxford 1987.

[23] S. Nosé, J. Chem. Phys. 81, 511 (1984).

[24] W.G. Hoover, Phys. Rev. A 31, 1695 (1985).

[25] Metal Reference Book, Ed. C.J. Smithells, Butterworths, London 1976. 